Kong. Res. J. 4(1) : 96-106, 2017

ISSN 2349-2694

Kongunadu Arts and Science College, Coimbatore.

\title{
CONSERVATION OF BIODIVERSITY AND SOCIO-CULTURAL DIMENSIONS OF SACRED GROVES OF KANNUR DISTRICT, KERALA
}

\author{
Sekaran, $\mathbf{S}^{1^{*}}$. and S. Nisha Raj ${ }^{2}$ \\ ${ }^{1} \mathrm{PG}$ and Research Department of Botany, Sree Narayana College, Kannur, Kerala. \\ ${ }^{2} \mathrm{PG}$ Department of Biotechnology, SAS,SNDP Yogam College, Konni, Kerala. \\ *E.mail: drsekhar72@gmail.com
}

\begin{abstract}
Sacred groves are small patches of forests, protected by local communities on religious grounds, rituals and culture. It represent a tradition of nature worship by dedicating patches of forests to deities and providing protection to such forest patches. They have immense value from genetic and ecological point of view. Results of studies conducted in eleven sacred groves in Kannur district of Kerala are compiled in this paper. Two hundred and three plant species including 10 true mangroves give an insight into the bioecological and socio cultural dimensions of sacred groves in helping and conserving the biological diversity. Kaliyattam a performing art with different forms of 'theyyam' is conducted every year offering to propitiate the deity by different communities in the villages. 'Devakooth' a theyyam performed by women in Thekkumpad kavu. is noteworthy among the performative rituals. These ritualistic practices centred around the sacred groves substantially contribute to the conservation and day- to-day management of ecological balance by sacred groves. The different types of roles played by sacred groves in maintaining the ecosystem integrity and biodiversity conservation are also presented in this paper.
\end{abstract}

Keywords: Biodiversity, Sacred groves, Kannur, Kerala.

\section{INTRODUCTION}

The socio-ecological system integrating with its cultural and spiritual dimensions with is still strong amongst many traditional societies. The initial impetus amongst traditional societies for conservation of biodiversity seems to have arisen out of the animistic religious belief system. The concept of "sacred grove" could be viewed as symbolic of "nature-human" inter connections with a variety of ritual linked to the diverse community living within the landscape boundary, who have their own right for natural resourse use, large communuty participation is ensured (Ramakrishnan, 2005). Sacred groves are patches of climax vegetations of past, reserved on religious grounds. They have immense value from genetical and ecological point of view. These are natural treasure houses of plants and animals that can satisfy the scientific, cultural, aesthetic needs of mankind.Traditional ecological knowledge and its functioning in India is a compled subject and least understood. Kerala is said to have about 360 groves which contain 660 plant species(Induchoodan, 1992).However it is estimated that in two Northern districts of Kerala,(Kannur,
Kasargod) there are more than 1000 groves (Unnikrishnan,1995). The kavu helps to conserve the diversity of plants and animals and also to build up and maintain cultural diversity of the region by providing platform for perfoming arts like "theyyam" and other festivals( Kunhikannan, 2005)

The presents study was carried out with to study the floristic diversity of certain sacred groves in Kannur and to elucidate the role of sacred groves in biodiversity conservation with sustainable livelyhood of traditional and cultural aspects.

\section{MATERIALS AND METHODS}

The presents study was carried out with to study the floristic diversity of eleven sacred groves in Kannur and to elucidate the role of sacred groves in biodiversity conservation with sustainable livelyhood of traditional and cultural aspects. The following sacred groves are selected for the study.

1) Madaikavu at Madai

2) Kunnathu Kavu, Pannenpara

3) Kizhakken Kavu, Chekkikulam

4) Muchilottu Kavu, Narathu

5) Neeliyar Kottam Kavu, Parassinikadavu 
6) Sri Thundikoth Bhagavathi Kshetram, Valiyannur

7) Valiavalappu Kavu, Thavakkara

8) Thalikkavu Sri Bhagavathi Kshethram, Thalikkavu

9) Kalarivathukal Bhagavathi Temple, Valapattanam

10) Aanthur Kavu, Parassinikadavu

11) Thekkumpad and Thaze kavu

Madaikavu is a famous sacred grove situated at Iripuram in Pazhayangadi about $20 \mathrm{~km}$ north of Kannur town. It is densely biodiverse region spreading about 10.5 acres. It is situated on the famous Madayi hills. Regarding the myths, the deity here is Madaikavilamma. Records suggest that this kavu was present even before $1300 \mathrm{AD}$. This is many centuries old and ancient records say that the Sri Rajarajeswara temple at Thaliparamba and Madayikavu are very closely related. The Kunnathu Kavu situated in Pannenpara the deity is Bhavur Karinkali, Kizhakkan Kavu is situated in Chekkikulam, Muchilottu Kavu is situated in Narathu, Neeliyar Kottam kavu is situated in Managattuparamba, Thundikoth Bhagavathi Kshetram is situated in Valiyanoor. The deity here is Bhagavathi, Valiyavalappu Kavu is situated in the middle of the town at Thavakkara. It is said to be more than 300 years old.

\section{Fig. 1 Selected Sacred Groves in Kannur}

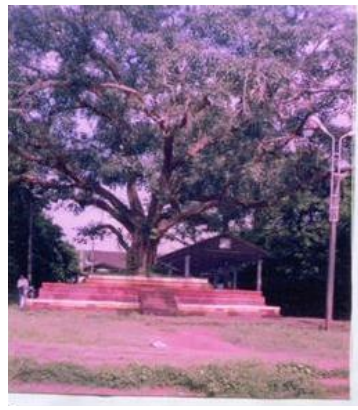

Madai Kavu

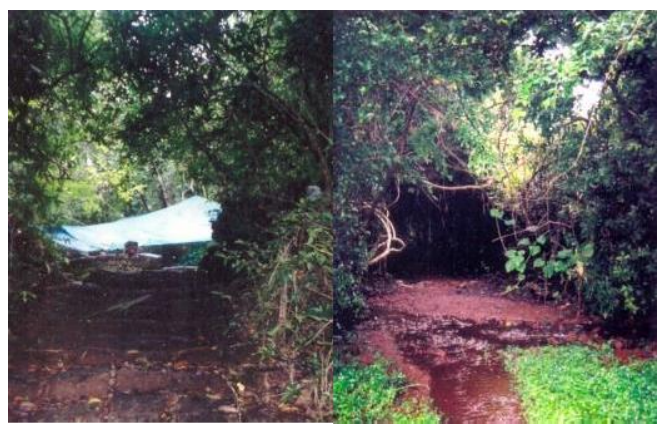

Neeliarkottam Kavu

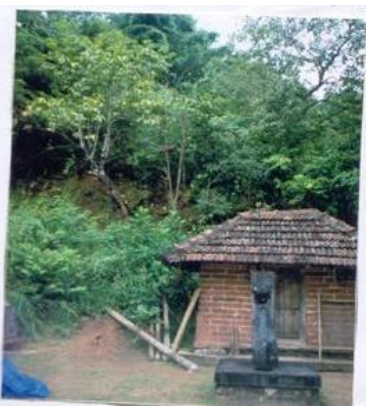

Aanthur Kavu

Madai vegetation

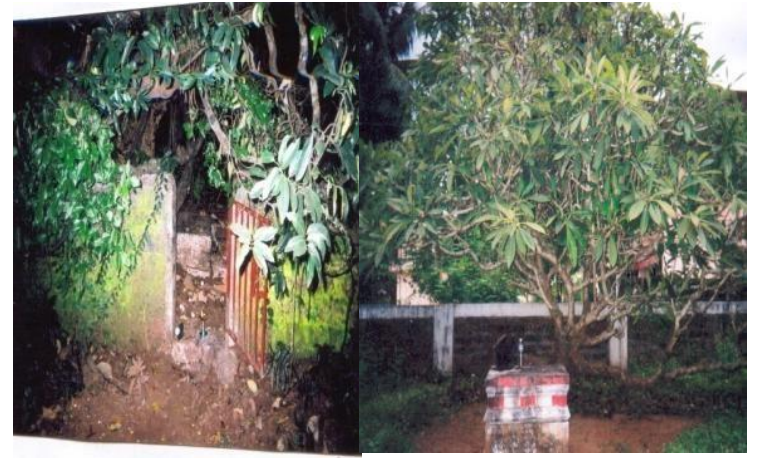

Thali kavu

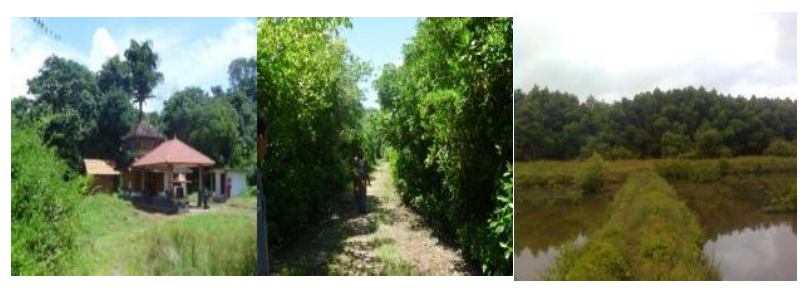

Thekkumpad kavu and Wetland s around the Sacred grove

Fig. 2. Believes and Conservation

(Rituals /Spirutuals)

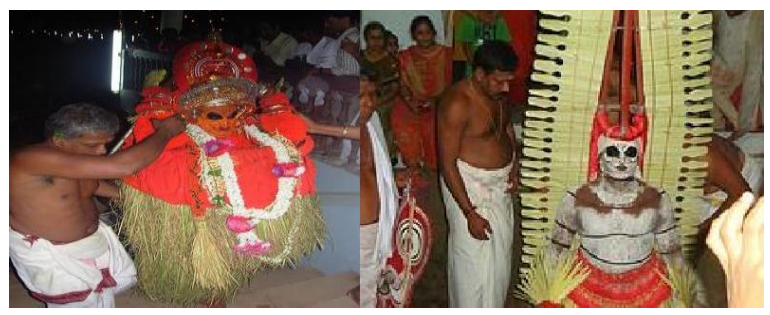

Theechamunndi

Gulikan

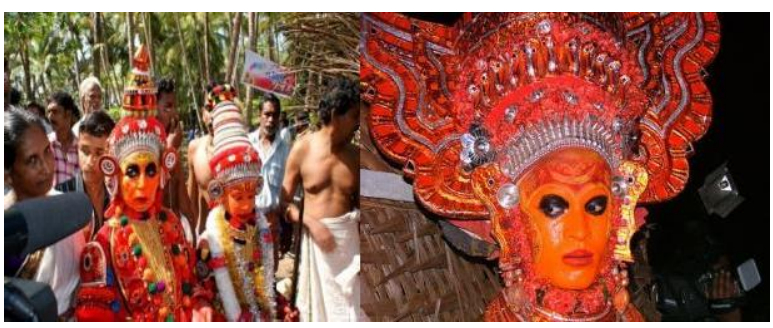

Devakkooth

Karanavar

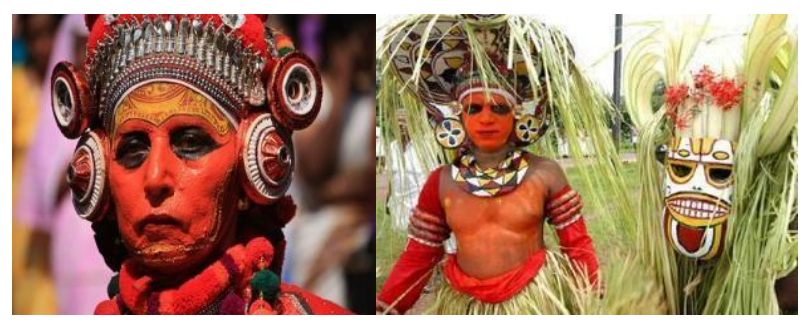

Devakkooth at Thekkumbad Maritheyyam at Madayi kavu

Thalikavu Sri Bhagavathi Kshetram. It is one of the oldest and still diverse sacred grove. It is situated at Thalikkavu in Kannur town itself. It is 
dated back to 250 years. Kalarivathukkal Bhagavathi Temple, Valapattanam,the 'Theyyam and Thira' festival of entire North Malabar is binded to this temple. It is a large sacred grove. Outside the sanctum sanatorium to the east is the Saraswathy Kshetram, to the north is the Nagakkavu and at the east is the great old banyan tree which is worshipped even today believing that the goddess had swing there. Aanthur Kavu situated nearby Parassinikadavu, the deity of this kavu is Bhagavathi.

Thekkumbadu village is a part of large cultural landscape within interconnections between the various ecosystem types, such as wetland ecosystems, water bodies, human management (kaippad paddy field) patch of forest around the sacred grove and mangrove forest ecosystem placed within a resource rich landscape unit provided the appropriate climate for sustaining protected forest ecosystem in the form of sacred groves(Thaye kavu), agro ecosystem(kaippad paddy cultivation between two kavu) and mangrove forest ecosystem.

The inhabitants around this wetland sacred grove of thekkumbadu maintain a spiritual connection and are integral part of there lives. The coastal marine ecosystems/wetland ecosystems are known to be productive, exceedingly valuable areas among the various biodiversity regions of the World. Living along the interface between land and sea, the mangrove ecosystem support genetically diverse groups of aquatic and terrestrial organisms.

\section{RESULTS AND DISCUSSION}

Kannur known as the land of looms and lores is famous for its rituals and custom based on ancestry which had played a lot in preserving the biodiversity at least to a small extent. Sacred groves are important among them. It helped a lot in Biodiversity conserved of the district directly and indirectly.

Table 1. List of plant species identified in Madaikavu, Madai.

\begin{tabular}{clcc}
\hline S.No & \multicolumn{1}{c}{ Botanical Name } & Family & Common Name \\
\hline 1 & Erythrina variegata L & Malvaceae & Murikku \\
2 & Glycerdia cepia & Fabaceae & Sheemakonna \\
3 & Ficus religiosa L. & Moraceae & Arrayal \\
4 & Aeglaia roxburchi Hiern & Meliaceae & Punyava \\
5 & Vernonia cinerea Less & Asteraceae & Poovamkurunthal \\
6 & Justicia japonica Thumb & Acanthaceae & Neelipoochedy \\
7 & Utricularia aurea Lour & Lentibulariaceae & Kakkapoovu \\
8 & Coleus umbonicus L. & Lamiaceae & Mathilkoorkka \\
9 & Justicia procumbens L. & Acanthaceae & \\
10 & Pouzolzia indica Gaud & Urticaceae & Neycheera \\
11 & Dalbergia volubilis Roxb. & Fabaceae & Jadavally \\
12 & Sopubia trifida Ham & Scrophularaceae & Kunhikolambi \\
13 & Justicia ekakusuma & Acanthaceae & Eakakusumam \\
14 & Heliotropium indicum L. & Boraginaceae & \\
15 & Neonotonia wightii Lackey & Fabaceae & Paraneelapoovu \\
16 & Rotala malambuzhansis & Lithraceae & Thadripoovu \\
17 & Vallisneria spiralis Linn. & Hydrocharitaceae & Ribbon plant \\
18 & Nymphoides krishnakesara & Nympheaceae & Pootthally \\
19 & Euphorbia kathragenesis & Euphorbiaceae & Paalutti \\
20 & Impatiens balsamina L. & Balsaminaceae & Kashithumba \\
21 & Zizyphus mauritiana (Lamk.) & Rhamnaceae & Vanthutali \\
22 & Rauvolfia serpentina & Apocyanaceae & Sarppagandhi \\
23 & Curcuma cannanorensis Ansari et al & Zingeberaceae & Kattumanghal \\
24 & Utricularia polygaloides Linn. & Urticaceae & Kakkpaoovu \\
25 & Lidernia ciliata (Colsm) & Scrophulariaceae & Chiravanakku \\
26 & Rotala malabaricum & Lythraceae & Parathamara \\
27 & Sapium insign Benth & Eurphorbiaceae & Kanmaram \\
28 & Strychnos rux.vomica L. & Loganiaceae & Kanhiram \\
29 & Curculego orchioides Gaertn & Hypoxidaceae & Nilappana \\
30 & Plumeria accuminata Ait. & Apocynaceae & Pala \\
31 & Sida rhombifolia L. & Malvaceae & Kurumthotty \\
\hline & & & \\
& & &
\end{tabular}




\begin{tabular}{llcc}
\hline 32 & Leucas plukenetii (Roth) Spreng. & Lamiaceae & Thumba \\
33 & Xanthium indicum Roxb & Asteraceae & Karamullu \\
34 & Phyllanthus amarus schum Th & Euphorbiaceae & Keezharnelly \\
35 & Lantana camara L & Lamiaceae & Kattuthulasi \\
36 & Physalis minima Linn. & Solanaceae & Muttambigha \\
37 & Cyclea peltata Diels & Pedaliaceae & Ellumpoovu \\
38 & Zehneria scabra Sond. & Cucurbitaceae & Kurukkanvellary \\
39 & Ipomaea sepiaria Koen & Convolvulaceae & Palamthangi \\
40 & Micrococca mercurialis Benth. & Eurphorbiaceae & Kunukady \\
41 & Anamirta cocculus W and A & Cucurbitaceae & Padolam \\
42 & Merremia tridentata L. Hall.f. & Convolvulaceae & Thiruthali \\
43 & Flacourtia montana Grah & Pittosporaceae & Karuvachakka \\
44 & Celosia argentea Linn. & Amaranthaceae & Mayoorasika \\
45 & Desmodium triflorum (L.) DC & Papilionaceae & Nilamparanda \\
46 & Smithia conferta J.E. Smith & Leguminosae & \\
47 & Memecylon malabaricum cogn & Melastomaceae & Kayamboo \\
48 & Amorphophallus dubius Bl. & Araceae & Chena \\
49 & Biophytum sensitivum DC & Gerahiaceae & Mukkutty \\
50 & Ericaulon & Ericaulaceae & Choothu \\
51 & Colocassia esculentum & Araceae & Thalu \\
52 & Oroxylum indicum (L.) Vent. & Bignoniaceae & Mothiravalli \\
53 & Plumbago zeylanica L. & Plumbaginaceae & Vellakoduveli \\
54 & Cryptolepis buchananii Roem.andSchult & Asclepidaceae & Palvalli \\
55 & Elephantopis scaber L. & Asteraceae & Aanachuvadi \\
56 & Justicia japonica Thunb. & Acanthaceae & Karimaram \\
57 & Evolvulus nummularis & Convolvulaceae & Vishnukranthi \\
58 & Buchanania augustifolia Roxb. & Anacardiaceae & \\
59 & Premna latifolia Roxb.) & Verbenaceae & Munja \\
60 & Vitex trifolia L. & Verbenaceae & Notchi \\
61 & Murdannia nudiflora (L.) Brenan & Commelinaceae & Paravellamkudiyan \\
62 & Ageratum conizoides $(L)$. & Asteraceae & Appakkadu \\
63 & Jatropha glandulifera Roxb. & Euphorbiaceae & Nattavanakku \\
\hline
\end{tabular}

Table 2. List of plant species identified in Kunnathu Kavu, Pannenpara.

\begin{tabular}{clcc}
\hline S.No & \multicolumn{1}{c}{ Botanical Name } & Family & Common Name \\
\hline 1 & Dioscoria alata. $\mathrm{L}$ & Dioscoreaceae & Kachhil \\
2 & Clerodendrum viscosum vent & Verbenaceae & Vattaperuvalam \\
3 & Mallotus philippinensis M.Arg & Euphorbiaccae & Kurukutty \\
4 & Piper nigrum L & Piperaceae & Thippali \\
5 & Naregamia alata wight and Arn. & Meliaceae & Nilanarakam \\
6 & Citrus grandis Osbeck & Rutaceae & Bambloose \\
7 & Cyclea peltata (Lamk) Hoof. F and Thoms & Menispermaceae & Padavally \\
8 & Wattakaka volubilis (L.f) stapf & Asclepiadiaceae & Palvally \\
9 & Lindernia ciliata Pennell & Scrophulariaceae & Chiravanakku \\
10 & Calycopteris floribunda (Roxb) Poir & Combretaceae & Pullanhi \\
11 & Caryota urens L & Arecaceae & Anappana \\
12 & Holigarna arnottiana Hook.f & Anacardiaceae & Cheru maram \\
13 & Plumeria rubra L. & Apocynaceae & Chembakam \\
14 & Cassia fistula L & Caesalpiniaceae & Konna \\
15 & Acacia auriculiformis L & Mimosae & Acacia \\
16 & Hibiscus rosasinensis L & Malvaceae & Chembarathy \\
17 & Santalum album L & Santalaceae & Chandanam \\
18 & Artocarpus heterophylla Lam. & Moraceae & Plavu \\
19 & Anacardium occidentale L & Anacardiaceae & Kashumavu \\
\hline
\end{tabular}




\begin{tabular}{clcc}
\hline 20 & Emilia sonchifolia L. DC & Asteraceae & Muyalcheviyan \\
21 & Phyllanthus amarus schum and Thonn & Euphorbiaceae & Kizharnelly \\
22 & Tectona grandis L.f & Verbenaceae & Jathi \\
23 & Curculigo orchioides Gaertn & Hypoxidaceae & Nilappana \\
24 & Ocimum sanctum L & Lamiaceae & Thulasi \\
25 & Alstonia scholaris L. R. Br. & Apocynaceae & Ezhilampala \\
26 & Costus speciosus (Koenig) smith & Zingiberaceae & Kanhipoovu \\
27 & Adenanthera pavonina L & Mimosae & Manjadi \\
28 & Pathos scandens L & Araceae & Paruvakodi \\
29 & Vigna vexillata L. A. Rich & Papilionaceae & Kattuzhunnu \\
30 & Mimusops elengi L & Sapotaceae & Elanghi \\
31 & Piper betle L & Piperaceae & Vettila \\
32 & Biophytum sensitivum DC & Oxalidaceae & Mukkutty \\
33 & Zehneria scabra Sond & Cucurbitaceae & Kurukkanvellary \\
\hline
\end{tabular}

Table 3. List of plant species identified in Kizhakken Kavu Chekkikulam.

\begin{tabular}{clcc}
\hline S.No & \multicolumn{1}{c}{ Botanical Name } & Family & Common Name \\
\hline 1 & Mussaenda frondosa L. & Rubiaceae & Vellila \\
2 & Hibiscus rosasinensis L. & Malvaceae & Chembarathy \\
3 & Ocimum sanctum Linn. & Lamiaceae & Thulasi \\
4 & Glycerdia cepia & Fabaceae & Sheemakonna \\
5 & Plumaria accuminata Ait. & Apocynaceae & Chembakam \\
6 & Ipomoea pentaphylla Jacq. & Convolvulaceae & Anchilachedi \\
7 & Lantana camara L & Verbenaceae & Aripoo \\
8 & Xanthium streumarium Wight & Asteraceae & Karamullu \\
\hline
\end{tabular}

Table 4. List of plant species identified in Muchilottu Kavu, Narathu.

\begin{tabular}{clcc}
\hline S.No & \multicolumn{1}{c}{ Botanical Name } & Family & Common Name \\
\hline 1 & Rauvolfia serpentina Benth & Apocynaceae & Sarppagandhi \\
2 & Zehneria scabra Sond. & Cucurbitaceae & Kurukkan vellary \\
3 & Carallia barachiata ( Lour.) Merr. & Rhizophoraceae & Vanghanna \\
4 & Chasalia curviflora Thw. & Rubiaceae & Velutha aval pori \\
5 & Santalum album L & Santalaceae & Chandanam \\
6 & Stachytarpheta urticaefolia (salisb.) sims. & Verbenaceae & Chiravanakku \\
7 & Justicia prostrata (cl.) Gamble & Acanthaceae & \\
8 & Lygodium fluxos smith & Pteridophyta & Polivally \\
9 & Quisqualis indica L & Combretaceae & Kulamarinhi \\
\hline
\end{tabular}

Table 5. List of plant species identified in Neeliyar Kottam Kavu, Parassinikadavu.

\begin{tabular}{clcc}
\hline S.No & \multicolumn{1}{c}{ Botanical Name } & Family & Common Name \\
\hline 1 & Alangium salvifolium Wang.var & Alangiaceae & Angholam \\
2 & Desmos lawii (Hook.f. and Thoms) & Anonaceae & Panal \\
3 & Artabotrys zeylanicus Hk.f. & Anonaceae & \\
4 & Knoxia wightiana wall & Rubiaceae & \\
5 & Jasminum ritchii Cl. & Oleiaceae & Kattupichhakam \\
6 & Memecylon lawsoni Gamb & Melastomaceae & \\
7 & Sonerila rheedii W.and A. & Melastomaceae & \\
8 & Strychnos nux- vomica L. & Loganiaceae & Kanhiram \\
9 & Bulbophyllum neilgherrense wight & Orchidaceae & Punnilachedi \\
\hline
\end{tabular}


Table 6. List of plant species identified in Sri Thundikoth Bhagavathi Kshetram,Valiyannur.

\begin{tabular}{|c|c|c|c|}
\hline S.No & Botanical Name & Family & Common Name \\
\hline 1 & Ocimum sanctum Linn & Lamiaceae & Thulasi \\
\hline 2 & Plumaria accuminata Ait & Apocynaceae & Chembakam \\
\hline 3 & Terminalia catappa Linn & Combretaceae & Badam \\
\hline 4 & Cassia fistula $\mathrm{L}$ & Caesalpiniaceae & Konna \\
\hline 5 & Mangifera indica L. & Anacardiaceae & Mavu \\
\hline 6 & Anacardium occidentale $\mathrm{L}$ & Anacardiaceae & Kashumavu \\
\hline 7 & Cleome viscose $\mathrm{L}$. & Capparidaceae & Kattukaduku \\
\hline 8 & Leucas plukenetti (Roth)Spreng. & Lamiaceae & Thumba \\
\hline 9 & Tridax procumbens $\mathrm{L}$. & Asteraceae & Thalatherippan \\
\hline 10 & Vernonia cinerea Less & Asteraceae & Poovamkurunthal \\
\hline 11 & Aerva lanata L. schult & Amaranthaceae & Cheroola \\
\hline 12 & Cyathula prostrata ( L.) Bl & Amaranthaceae & Aanachuvadi \\
\hline 13 & Mitracarpus verticillatus(schum and Thonn) & Rubiaceae & - \\
\hline 14 & Mimosa pudica L & Mimosae & Thottavady \\
\hline 15 & Sida rhombifolia $\mathrm{L}$. & Malvaceae & Kurumthotty \\
\hline 16 & Phyllanthus amarus Schum and Thonn & Euphorbiaceae & Keezharnelly \\
\hline 17 & Scoparia dulcis L. & Scrophulariaceae & Kallurukky \\
\hline 18 & Gloriosa superba L. & Liliaceae & Mendhonni \\
\hline 19 & Cassia mimosoidis L. & Caesalpiniaceae & - \\
\hline 20 & Crotolaria striata DC. & Fabaceae & Kilukkampatti \\
\hline 21 & Hedyotis diffusa willd & Rubiaceae & \\
\hline 22 & Portulaca oleracea L. & Portulacaceae & pathumanipoov \\
\hline 23 & Ludwigia hyssopifolia (G.Don)Exell & Onagraceae & - \\
\hline 24 & Memecylon umbellatum Burm.F. & Melastomaceae & Kayambu \\
\hline
\end{tabular}

Table 7. List of plant species identified in Valiavalappu Kavu, Thavakkara.

\begin{tabular}{|c|c|c|c|}
\hline S.No & Botanical Name & Family & Common Name \\
\hline 1 & Amaranthus irridis L. & Amaranthaceae & Mullancheerra \\
\hline 2 & Sesamum indicum $\mathrm{L}$. & Pedaliaceae & Kattellu \\
\hline 3 & Carica papaya Linn. & Caricaceae & Papaya \\
\hline 4 & Mukia maderaspatana L. Roem. & Cucurbitaceae & Kattuvellary \\
\hline 5 & Ageratrum conyzoides L. & Asteraceae & Appa \\
\hline 6 & Colocasia esculenta $\mathrm{L}$. & Araceae & Kattu chembu \\
\hline 7 & Plumaria accuminata Ait. & Apocynaceae & Chembakam \\
\hline 8 & Tamarindus indicus L. & Caesalpiniaceae & Puli \\
\hline 9 & Strychnos nux-vomica L. & Loganiaceae & Kanhiram \\
\hline 10 & Leucas blukenetii (Rosh.) Spreng. & Lamiaceae & Thumba \\
\hline 11 & Tagetus erectus & Asteraceae & Chetty \\
\hline 12 & Hyptis suaveolens (L.) Poir & Lamiaceae & Kattuthulasi \\
\hline 13 & Calotropis gigantea R.Br. & Asclepiadaceae & Erikku \\
\hline 14 & Sida rhombifolia $\mathrm{L}$. & Malvaceae & Kurumthotty \\
\hline 15 & Anona squamosa L. & Anonaceae & Aathachakka \\
\hline 16 & Curculigo orchioides Gaertn & Hypoxidaceae & Nilappana \\
\hline 17 & Adhatoda vasica Nees. & Acanthaceae & Adalodakam \\
\hline 18 & Desmodium trifolium L. DC. & Leguminosae & Nilamparanda \\
\hline 19 & Macaranga peltata (Roxb.) Huell Arg. & Euphorbiaceae & Uppila \\
\hline 20 & Crotalaria striata DC. & Papilionaceae & Kilukilukkipoo \\
\hline 21 & Lantana camara L. & Verbenaceae & Arippoo \\
\hline 22 & Ficus carica & Moraceae & Atthi \\
\hline 23 & Datura metel L. & Solanaceae & Ummam \\
\hline 24 & Ocimum sanctum Linn. & Lamiaceae & Krishnathulasi \\
\hline 25 & Cassia tora L. & Caesalpiniaceae & Thavara \\
\hline
\end{tabular}


Table 8. List of plant species identified in Thalikavu Sri Bhagavathi Kshetram Thalikavu.

\begin{tabular}{|c|c|c|c|}
\hline S.No. & Botanical Name & Family & Common Name \\
\hline 1 & Plumeria acuminata Ait. & Apocynaceae & Chembakam \\
\hline 2 & Strychnos nux-vomica L. & Loganiaceae & Kanhiram \\
\hline 3 & Ficus religiosa L. & Moraceae & Arayal \\
\hline 4 & Aegle marmelose corr & Rutaceae & Koovalam \\
\hline 5 & Cassia fistula L. & Caesalpiniaceae & Konna \\
\hline 6 & Sida rhombifolia L & Malvaceae & Kurumthotty \\
\hline 7 & Crotalaria pallida Dryand & Papilionaceae & Kilukilukkipoovu \\
\hline 8 & Gloriosa superba L. & Liliaceae & Mendhonni \\
\hline 9 & Hyptis suaveolens L. Poir & Lamiaceae & Kattuthulasi \\
\hline 10 & Leucas plukenetii (Roth) spreng & Lamiaceae & Thumba \\
\hline 11 & Mimosa pudica L. & Mimosae & Thottavadi \\
\hline 12 & Datura metel L. & Solanaceae & Ummam \\
\hline 13 & Cleome viscosa Linn & Capparidaceae & Kattukadugu \\
\hline 14 & Macaranga peltata (Roxb.) Muell-Arg. & Euphorbiaceae & Uppila \\
\hline 15 & Rauvolfia serpentina (L) Kurz & Apocynaceae & Sarppaghandhi \\
\hline 16 & Curculigo orchioides Gaertn. & Hypoxidaceae & Nilappana \\
\hline 17 & Mussaenda frondosa $\mathrm{L}$. & Rubiaceae & Veleela \\
\hline 18 & Caryota urens L. & Arecaceae & Aanapanna \\
\hline 19 & Zizyphus oenoplia L. Mill & Rhamnaceae & Kottakapazham \\
\hline 20 & Spondias pinnata L.f. kurz. & Anacardiaceae & Ambayam \\
\hline 21 & Tabernaemontana heyneana. Wall & Apocynaceae & Pala \\
\hline 22 & Pouzolzia wightii Benn-Pl. Jan Rar & Urticaceae & Neycheera \\
\hline 23 & Cassia tora L. & Caesalpiniaceae & Thavara \\
\hline 24 & Colocassia esculenta L.Schotta & Araceae & Thalu \\
\hline 25 & Ocimum sanctum Linn. & Lamiaceae & Krishnathulasi \\
\hline 26 & Ageratum conyzoides L. & Asteraceae & Appa \\
\hline 27 & Urena lobala L. & Malvaceae & Uthiram \\
\hline 28 & Uvaria narum (Dunal) Wall. & Anonaceae & Nagavalli \\
\hline 29 & Jasminum multiflorum Burm.f & Oleaceae & Kattumulla \\
\hline 30 & Tagetus erectus & Asteraceae & Chetty \\
\hline
\end{tabular}

Table 9. List of plant species identified in Kalarivathukal Bhagavathi Kshetram, Valapattanam.

\begin{tabular}{clcc}
\hline S.No. & \multicolumn{1}{c}{ Botanical Name } & Family & Common Name \\
\hline 1 & Pothos scandens L. & Araceae & Paruvakodi \\
2 & Mallotus philippensis (Lam.) Muelt Arg. & Euphorbiaceae & Koovukoodi \\
3 & Calycopteris floribunda (Roxb.) Poin & Combretaceae & Jadapoovu \\
4 & Strychnos nux-vomica L. & Loganiaceae & Kanhiram \\
5 & Alstonia scholaris L.R.Br. & Apocynaceae & Ezhilampala \\
6 & Phyllanthus emblica Schum Th & Euphorbiaceae & Nelli \\
7 & Caryota urens L. & Arecaceae & Anappana \\
8 & Cassia occidentalis L. & Caesalpiniaceae & May flower \\
9 & Santalum album L. & Santalaceae & Chandanam \\
10 & Dalbergia volubilis Roxb. & Fabaceae & Jadavally \\
11 & Plumeria acuminata Ait. & Apocynaceae & Chembakam \\
12 & Quisqualis indica L. & Combretaceae & Kulamarinhi \\
\hline
\end{tabular}

Table 10. List of plant species identified Aanthur Kavu, Parassinikadavu.

\begin{tabular}{clcc}
\hline S.No & \multicolumn{1}{c}{ Botanical Name } & Family & Common Name \\
\hline 1 & Calyopteris floribunda (Roxb.) Poir. & Combretaceae & Jadapoovu \\
2 & Strychnos nux-vomica L. & Loganiaceae & Kanhiram \\
3 & Mussaenda frondosa L. & Rubiaceae & Veleela \\
4 & Plumeria accuminata. Ait. & Apocynaceae & Chembakam \\
\hline
\end{tabular}




\begin{tabular}{clcc}
\hline 5 & Aegle marmelos corr. & Rutaceae & Koovalam \\
6 & Ficus religoisa L. & Moraceae & Arayal \\
7 & Curculigo orchioides Gaertn. & Hypoxidaceae & Nilappana \\
8 & Caryota urens L. & Arecaceae & Aanappana \\
9 & Azadirachta indica A Juss & Miliaceae & Veppu \\
10 & Santalum album L. & Santalaceae & Chandam \\
11 & Tectona grandis L.f. & Verbenaceae & Jadhi \\
12 & Holigarna arnottiana Hook.f. & Anacardiaceae & Cheru maram \\
13 & Alstonia scholaris (L.) R. Br. & Apocynaceae & Ezhilampala \\
14 & Ocimum sanctum Linn. & Lamiaceae & Thulasi \\
15 & Emilia sonchifolia (L.) DC & Asteraceae & Muyal cheviyan \\
16 & Cassia fistula L. & Caesalpiniaceae & Konna \\
17 & Anona squamosa Linn. & Anonaceae & Aathachakka \\
18 & Sida rhombifolia L. & Malvaceae & Kurumthotty \\
19 & Jasminum muttiflorum Burm.f. & Oleaceae & Kattumulla \\
20 & Memecylon umbellatum Burm.f. & Melastomaceae & Kashavu \\
21 & Mimusops elengi Linn & Sapotaceae & Elanghi \\
\hline
\end{tabular}

Table 11. Plants collected from Thekkumbad (Mangrove Associated Plants).

\begin{tabular}{|c|c|c|c|c|}
\hline S.no & Botanical name & Family & Local name & Habit \\
\hline 1 & Utricularia reticulate J. E.Sm & Lentibulariaceae & Kakkapoovu & Herb \\
\hline 2 & Cyanotis cristata (L.) D.Don & Commelinaceae & & Herb \\
\hline 3 & Ipomoea pes-caprae (L.) Sweet & Convolvulaceae & & Herb \\
\hline 4 & Cyperus rotundus L.Sp. Pl & Cyperaceae & Muthanga & Herb \\
\hline 5 & Carex filicina Nees & Cyperaceae & & Herb \\
\hline 6 & Clerodendrum inerme (L.) & Verbenaceae & & Shrub \\
\hline 7 & Eicchornia crassipes (Mart.) Solms & Pontederiaceae & & Herb \\
\hline 8 & Sphaeranthus indicus L. & Asteraceae & & Herb \\
\hline 9 & Exacum tetragonum Roxb. Fl & Gentianaceae & & Herb \\
\hline 10 & Ludwigia octovalvis (Jacq.)Raven & Onagraceae & & Herb \\
\hline 11 & Wedelia biflora (L.) DC & Asteraceae & Kammal poovu & Herb \\
\hline 12 & Premna serritifolia $L$. & Verbenaceae & & Shrub \\
\hline 13 & Cassia mimosoides L. & Caesalpiniaceae & & Herb \\
\hline 14 & Scirpus dichotoma $L$. & Cyperaceae & & Herb \\
\hline 15 & Eclipta alba prostrata (L.)L.Mant & Asteraceae & Kayyoonji & Herb \\
\hline 16 & Ageratum conyzoides $L$. & Asteraceae & & Herb \\
\hline 17 & Lindernia crustacean (L.)F.v-Muell & Scrophulariaceae & & Herb \\
\hline 18 & Limnophila indica (L.)Druce & Scrophulariaceae & & Herb \\
\hline 19 & Commelina benghalensis $L$. & Commelinaceae & Vazhapadathi & Herb \\
\hline 20 & Desmodium triflorum (L.) & Papilionaceae & & Herb \\
\hline 21 & Desmodium laxiflorum Dc & Papilionaceae & & Herb \\
\hline 22 & Oldenlandia repens $L$. & Rubiaceae & & Herb \\
\hline 23 & Derris trifoliata Lour.Fl. & Papilionaceae & & $\begin{array}{c}\text { Climbing } \\
\text { shrub }\end{array}$ \\
\hline 24 & Eriocaulon dianae Fyson var. & Eriocaulaceae & & Herb \\
\hline 25 & Crotalaria juncea $L$. & Papilionaceae & & Herb \\
\hline 26 & Scoparia dulcis $L$. & Scrophulariaceae & Kallurukki & Herb \\
\hline 27 & Synedrella nodiflora $L$. & Asteraceae & & Herb \\
\hline 28 & Vernonia arboriea Buch -Ham & Asteraceae & Poovakurundal & Herb \\
\hline 29 & Sida acuta Burm.f.Fl & Malvaceae & Kurundhotti & Herb \\
\hline 30 & Leucas aspera (Wild.)Link & Lamiaceae & Thumba & Herb \\
\hline 31 & Gloriosa superb $L$. & Liliaceae & Mendonni & Climber \\
\hline 32 & Vanda roxburghii $R \cdot B r$ & Orchidaceae & Maravazha & Herb \\
\hline 33 & Ixora coccinea $L$. & Rubiaceae & Kattu thechi & Shrub \\
\hline
\end{tabular}




\begin{tabular}{|c|c|c|c|c|}
\hline 34 & Lantana camara L. & Verbenaceae & Arippu & Shrub \\
\hline 35 & Justicia simplex D.Don & Acanthaceae & & Herb \\
\hline 36 & Mimusops elengi $L$. & Sapotaceae & Elangi & Tree \\
\hline 37 & Ficus religiosa $L$. & Moraceae & Arayal & Tree \\
\hline 38 & Costus speciosus (koen.) Smith & Zingiberaceae & & Herb \\
\hline 39 & Mukia maderaspatana (L.) & Cucurbitaceae & Kurukan vellari & Climber \\
\hline 40 & Erythrina variegate Lam. & Papilionaceae & Murikke & Tree \\
\hline 41 & Bacopa monnieri (L.) & Scrophulariaceae & Brammi & Herb \\
\hline 42 & Tinospora cordifolia (Thunb.) Miers & Menispermaceae & Chittamrudh & Climber \\
\hline 43 & Arenga wightii Griff & Arecaceae & Kaattupana & Tree \\
\hline 44 & Bombax ceiba L. & Bombacaceae & Theepetimaram & Tree \\
\hline 45 & Holigarna arnottiana Hook. & Anacardiaceae & Cheru maram & Tree \\
\hline 46 & Thespesia populnea (L.) & Malvaceae & Puvarasu & Small tree \\
\hline 47 & Melastoma malabathricum $L$. & Melastomataceae & Athiraani & Shrub \\
\hline 48 & Cinnamomum zeylanicum Garc. & Lauraceae & Karuvapatta & Tree \\
\hline 49 & Calophyllum inophyllum L. & Clusiaceae & Attupunnam & Tree \\
\hline 50 & Morinda citrifolia $L$. & Rubiaceae & Manjanathi & Tree \\
\hline 51 & Cerbera manghas & Apocynaceae & Othalom & Small tree \\
\hline 52 & Aerva lanata (L.) & Amaranthaceae & Cherupula & Herb \\
\hline 53 & Pandanus fascicularis $L$. & Pandanaceae & Kaitha & Small tree \\
\hline 54 & Ipomoea companulata $L$. & Convolvulaceae & Manippoovu & Climber \\
\hline 55 & Glyricidea sepia (Jacq.)Walp. & Papilionaceae & Seemakonna & Tree \\
\hline 56 & Ananas comosus Mill. & Bromeliaceae & Kaitha chakka & Shrub \\
\hline 57. & Calamus rotang $L$. & Arecaceae & Chooral & Shrub \\
\hline 58 & Vitis trifolia $L$. & Vitaceae & Kaatumunthiri & Climber \\
\hline 59. & Ocimum sanctum $L$. & Lamiaceae & Thulasi & Herb \\
\hline 60. & Hyptis suaveolens $L$. & Lamiaceae & Katuthulasi & Herb \\
\hline 61. & Heliotropium indicum $L$. & Boraginaceae & & Herb \\
\hline 62. & Rauvolfia serpentina & Apocynaceae & Sarpagandhi & Herb \\
\hline 63. & Alstonia constricta & Apocynaceae & Pala & Tree \\
\hline 64 & Dioscoria alata & Dioscoriaceae & Kaachil & Climber \\
\hline 65. & Eupatorium odaratum & Asteraceae & Communistpacha & Shrub \\
\hline 66. & Colacasia esculenta & Araceae & Chembu & Herb \\
\hline 67. & Euphorbia hirta & Euphorbiaceae & & Herb \\
\hline 68. & Biophytum sensitvum & Oxalidaceae & Mukutti & Herb \\
\hline 69. & Tectona grandis L.f. & Verbenaceae & Teak & Tree \\
\hline 70. & Melochia corchorifolia $L$. & Sterculiaceae & Mullan chedi & Herb \\
\hline 71. & Coix lacremajobi & Poaceae & Kalla chedi & Herb \\
\hline 72. & Alysicarpus vaginalis & Papilionaceae & & Herb \\
\hline
\end{tabular}

Table 12. True Mangrove plants collected from Thekkumpad.

\begin{tabular}{clccc}
\hline S.no & \multicolumn{1}{c}{ Botanical name } & Family & Local name & Habit \\
\hline 1 & Acanthus ilicifolius L. & Acanthaceae & Chulli & Shrub \\
2 & Aegiceras corniculata L. & Myrsinaceae & Chakkara kandal & Tree \\
3 & Avicennia marina Fosk)vierh & Avicenniaceae & Uppatti & Tree \\
4 & Avicennia officinalis L. & Avicenniaceae & Oori & Tree \\
5 & Bruguiera cylindrical $(L) B l$ & Rhizophoraceae & & Tree \\
6 & Exoecaria agallocha L. & Euphorbiaceae & Kannampotti & Tree \\
7 & Kandelia candel (L)Druce & Rhizophoraceae & Poo kandal & Tree \\
8 & Rhizophora mucronata.Lamk & Rhizophoraceae & Pranthan kandal & Tree \\
9 & Rhizophora apiculata Blume & Rhizophoraceae & & Tree \\
10 & Sonneratia alba J.Smith & Sonneratiaceae & Nakshatra kandal & Tree \\
\hline
\end{tabular}


Table 13. Cultivated crops.

\begin{tabular}{clccc}
\hline S.no & \multicolumn{1}{c}{ Botanical name } & Family & Local name & Habit \\
\hline 1 & Oryza sativa L. & Poaceae & Nellu & Herb \\
2 & Musa paradisiacal $L$. & Musaceae & Vaazha & Gigantic herb \\
3 & Cocos nusifera L. & Arecaceae & Thengu & Tree \\
4 & Ipomoea batatas. $L$ & Convolvulaceae & Madura kizhangu & Trailing herb \\
5 & Zingiber officinalis Rose. & Zingiberaceae & Injhi & Herb \\
6 & Maranta arundinaaceae $L$. & Marantaceae & Koova & Herb \\
7 & Manihot esculenta & Euphorbiaceae & Maracheeni & Shrub \\
8 & Cucumis sativus $L$. & Cucurbitaceae & Vellari & Climber \\
9 & Lathyrus sps. & Papilionaceae & Kota payar & Climber \\
10 & Trichosanthes anguina $L$. & Cucurbitaceae & Padavalam & Climber \\
\hline
\end{tabular}

In the present study eleven important sacred groves are selected in Kannur district. The floristic diversity of selected ten sacred groves shows 151 of plant species and 131 genera belong to 56 families were reported in ten sacred groves. Among the these in Madayi kavu reported 63 plant species, followed by Kunnathu kavu 33 species, Thalikavu 30 species, Valiavalappu kavu 25 species, Thundikoth Bhagavathi Kshetram 24 species,Aanthur kavu 21 species, Kalarivathukal Bhagavathi Temple 12 species, Muchilottu kavu and Neeliarkottam kave recorded 9 species each and, Kizhakken kavu recorded only 8 species. The kavu helps to conserve diversity of plants and animals and also to build up and maintain cultural diversity of the region by providing platform for performing arts like"theyyam" and other festivals (Kunhikannan, 2005). The list plants collected from these scred groves are presented in Table1-10.

The Thekkumpad kavu has the unique nature compared to the above ten sacred grove In the present study, 92 plant species identified, of these 92 species, 72 species are mangrove associates and 10 species are true mangroves. The list plants collected from these scred groves are presented in Table11-14. The recorded true mangroves are Acanthus ilicifolius. L, Aegiceras corniculata L, Avicennia marina (Forsk)Vierh, Avicennia officinalis L, Avicennia alba Blume, Bruguiera cylindrical (L) Bl, Bruguiera gymnorhiza L, Exoecaria agallocha L, Kandelia candel (L) Druce, Rhizophora mucronata Lamk, Rhizophora apiculata and Sonneratia alba J.Smith.Ten species are identified as cultivated plants by the local peoples. These 92 plant species belongs to the 48 family and 87 genera. The herbaceous plants are dominant in Thekkumpad and 47 herbaceous plants are identified. Trees, shrubs and climbers are also recorded. 25 trees identified among these 3 of them are small trees. Besides this 11 shrubs and 9 climbers are noticed. Asteraceae, paplionaceae are found dominant families in which 7 genera each reported in these families. Among the mangrove associates Cyperaceae plants are dominant.Indeed many such species that are culturally valued ecologically significant keystone value to the ecosystem, through their contribution to ecosystem integerity (Ramakrishnan et al 1998, 2000). The avifauna of the certain sacred groves of North Malabar including Thazekkavu of thekkumpad were reported (Sasikumar 2005)

The present study give an insight into the bio-ecological and socio cultural dimensions of sacred groves in helping and conserving the biological diversity. Kaliyattam a performing art with different forms of 'theyyam' is conducted every year offering to propitiate the deity by different communities in the villages. These festivals are socio-religious ceremonies performed in north Kerala since ancient time (Kurup,2004). The 'Devakooth' a theyyam performed by women in Thekkumpad kavu. is noteworthy among the performative rituals. These ritualistic practices centred around the sacred groves substantially contribute to the conservation and day- to-day management of ecological balance by sacred groves.Maritheyyam will be performed at Madayi kavu on the $16^{\text {th }}$ day of Malayalam month karkidagam.

Sacred groves are the part of the socioecological system,the concept of village as part of a large cultural landscape, with interconnections between the various ecosystem type such as forests, water bodies and human-managed agroecosystem types placed within a resource rich landscape unit provideed the appropriate climate for sustaining protected forest ecosystems in the form of sacred groves.In other words, presence of resource rich healthier natural ecosystem type is a prerequisite for conservation of the sacred groves. 


\section{REFERENCES}

Induchoodan, N.C. (1992). Ecologicla studies on the scred groves of kerala. Final report to WWF, New Delhi

Kunhikannan, C. (2005). Traditions, Rituals and Biodiversity in Sacred grove of Karakkakavu, kasargod district, Kerala state-a case study. In: C. Kunhikannan and B. Gurudev Singh (eds.) Strategy for Conservation of Sacred Groves. Institute of Forest Genetics and Tree Breeding, Coimbatore, India. pp 125-136.

Kurup, K.K.N. (2004). Theyyam-A Ritual Dance of Kerala http://www.theyyam.com.

Ramakrishnan, P.S., (2005). Sacred Groves Integrated within the Cultural landscape.In:C.Kunhikannan and B. Gurudev Singh (eds) Strategy for Conservation of Sacred Groves. Institute of Forest Genetics and Tree Breeding, Coimbatore, India. p. 108-116.
Ramakrishnan, P.S., Saxena, K.G and Chandrasekara, (eds.) (1998). Conserving the Sacred: For Biodiversity Management. UNESCO and Oxford and IBH Publ.New Delhi. p. 480.

Ramakrishnan, P.S., U.M. Chandrasekara, C. Elourd, C.Z. Guilmoto, R.K. Maikhuri, K.S. Rao, S. Sankar and K.G. Saxena, (2000). Mountain Biodiversity, Land Use Dynamics and Traditional Ecological Knowledge. UNESCO and Oxford and IBH Publ.New Delhi. p. 353.

Sasikumar, C. (2005). Avifauna of the sacred groves of North Kerala. In: C.Kunhikannan and B. Gurudev Singh (eds) Strategy for Conservation of Sacred Groves. Institute of Forest Genetics and Tree Breeding, Coimbatore, India. p. 97107.

Unnikrishnan, E. (1995). Uttarakeralathile Visudhavanangal oru Paristhiti- Nadodiamsekarapadanam (Sacred groves of North Kerala- an Eco-folklore study) - Malayalam, Jeevarekha, Thrissur . Kerala. 